\title{
Automatic Correction of the 3D Orientation of the Brain Imagery
}

\author{
Xin Liu ${ }^{1}$ Celina Imielinska ${ }^{1}$ S. Connolly $\mathrm{Jr}^{2}$ A. D' Ambrosio ${ }^{2}$ \\ ${ }^{1}$ Department of Biomedical Informatics \\ ${ }^{2}$ Dept of Neurological Surgery \\ Columbia University, New York, NY.
}

\begin{abstract}
Classification of human brain pathologies can be guided by the estimation of the departure of $3 D$ internal structures from the normal bilateral symmetry. However symmetry based analysis can 't be precisely carried out when the $3 D$ brain orientation is misaligned, a common occurrence in clinical practice. In this paper, a technique to automatically identify the symmetry plane and correct the $3 D$ orientation of volumetric brain images in a cost effective way is developed. The algorithm seeks the best sampling strategies to realign $3 D$ volumetric representation of the brain within scanner coordinate system. The inertia matrix is computed on the sampled brain, and the principle axes are derived from the eigenvectors of the inertia matrix. The technique is demonstrated on MR and CT brain images and the detected symmetry plane that is orthogonal to the principle vectors is provided. A spatial affine transform is applied to rotate the 3D brain images and align them within the coordinate system of the scanner. The corrected brain volume is re-sliced such that each planar image represents the brain at the same axial level.
\end{abstract}

Keywords-Brain symmetry, Principle Component Analysis, 3D symmetry, inertia matrix.

\section{INTRODUCTION}

Detection and classification of human brain pathologies can be guided by the estimation of the departure of $3 \mathrm{D}$ internal structures from the normal bilateral symmetry. This comparison is useful since the human brain presents high level of bilateral symmetry that is partially absent under (asymmetric) pathological conditions. Symmetry is used by both clinical experts and automatic diagnostic systems to detect qualitatively asymmetric pattern indicating a wide range of pathologies (tumor, stroke, bleeding). However symmetry based analysis is hindered when the 3D brain orientation is misaligned, a common occurrence in clinical practice, since the misalignment causes the plane to intersect the symmetric structures at different heights. For neuro-radiologists and computer program to correctly assess the pathological asymmetries it is crucial that the neural scans are registered to the coordinate system of the scanner. Lack of such registration may results in the images being inhomologous within the same coronal or axial level.

The detection and computation of the symmetry plane is a necessary step in symmetry-based analysis of neural images. Assessment by either a clinical expert and/or automatic system based on symmetry analysis (for example the Relative Difference Map (RDM) quantification [3]) must account for the realignment of the brain to the scanner coordinate system.

The paper is organized as follows. In section 2, we overview existing methods. The algorithm is described and discussed in section 3 . The results are presented in section 4 and conclusions are provided in section 5 .

\section{BACKGROUND}

The normal brain exhibits approximate bilateral symmetry with respect to mid-sagittal plane and it is useful to define the mid-sagittal plane as the plane best separates the brain into two bi-fold mirror hemispheres. Approaches to estimate the mid-sagittal plane can be placed into two major categories: 2D based methods; and 3D based methods. 2D methods that are applied to individual 2D scans, mot always can be generalized to $3 \mathrm{D}$ cases. For example, Brummer [4] proposes a method of using the Hough transform to identify cerebral inter-hemispheric fissure. Marais [10] extracts the fissure using snakes, and uses an orthogonal regression from a set of control points. The method presented by Liu [7], sequentially deals with 2D slices for finding symmetry axis for each coronal or axial slice, and then computes a 3D plane from set of these lines. Because these methods process brain volume sliceby-slice, the global symmetry of the whole brain may not be captured. In case where the head is tilted along y axes, as in Fig.1, a structure displayed in the same axial slice will not reside in the same plane and the symmetry axes computed independently in each slice will produce flawed final result.

In 3D based approaches the plane that maximizes the bilateral symmetry is captured. Prima et al [2] computes local similarity measures between two sides of the brain, using block matching procedure [6]. Ardekani [5], conducts iterative search on the unit sphere, in order to find the plane with respect to which the image exhibits maximum symmetry. These algorithms that are based on local search reduce the amount of computation, but fail on clinical brain images with gross asymmetries often caused by pathological conditions. 


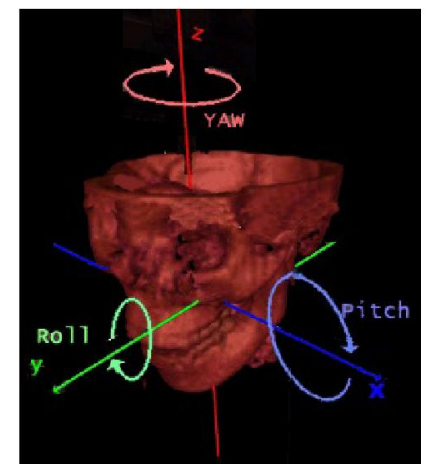

Fig. 1 The Pitch, Roll and Yaw angle of the brain. The $z$ direction is assumed to be aligned with the major axis of the scanner. The $x$ direction is chosen as left to right, and $y$ axes is chosen as posterior to anterior directions.

In this paper, a technique to automatically identify the symmetry plane and correct the 3D orientation of volumetric brain images in a cost effective way is developed. It is a $3 \mathrm{D}$ based method incorporating head extraction, the principle component analysis [2] and reinterpolation. We borrow the following assumptions from the work of Minovic et al [8].

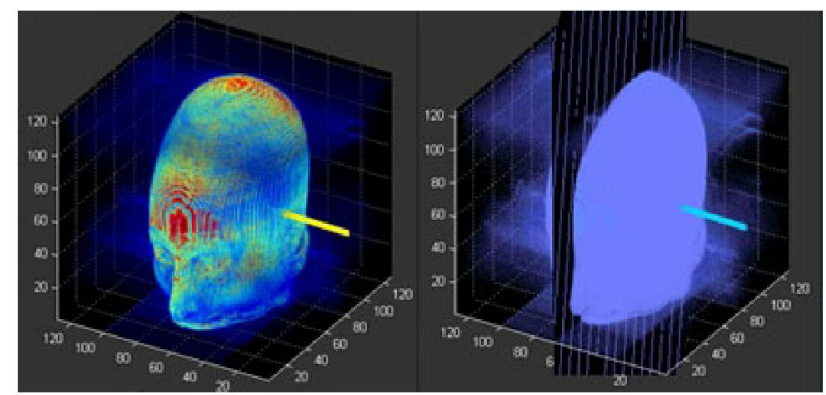

(a)

(b)

Fig. 2. (a) Illustration of one principle axis (the yellow vector) in volume rendered MRI images. (b) the demonstration of the relationship between the symmetry plane and the principle axis. In brain images, the mid-sagittal plane is orthogonal to the principle axis whose associated eigenvector has the smallest eigenvalue.

- Any plane of symmetry in a body is orthogonal to a principal axis;

- Any axis of symmetry in a body is a principle axis

Inspired by Minovic's work, Sun [6] developed an algorithm for finding symmetry planes of 3D objects using Gaussian image representation. Their work focuses on synthesized objects, that is not necessarily suitable to handle complex geometry of radiological brain images and all other problems related to the errors generated during the acquisition process.

The principle axis based method [2], can potentially fail to solve the problem of brain symmetry because, abnormal asymmetries distort the underlying symmetry of the brain. However, it is our belief that such pathological asymmetries only distort local symmetry, without altering the global geometry of the rigid body. Therefore, the $3 \mathrm{D}$ orientation of brain should remain unaffected. By treating the head as a solid $3 \mathrm{D}$ object, we can provide a robust method to compute successfully the plane of symmetry, and making it resistant to any specificity of brain internal geometry.

\section{ALGORITHM OVERVIEW}

We assume an idealized situation, with the diameter of all three principle axes of the human brain to be distinctive, and that we have a set of slices representing a complete head volume. Thus, neither the top of the head is truncated, nor too big sections of the neck and shoulder are present, as in Fig.2. Under those assumptions, our algorithm uses the following operations:

Step 1) We subtract the background from the image and make the tilted volume $V_{t}$ as a binary volume $B_{t}$. Then we remove all the slices that do not enclose head-only (the neck and shoulders if applicable). By doing this, we guarantee that our binary volume $B_{t}$ is a 3 dimensional ellipsoid-like shape, where there exists 3 distinctive principle axes;

$$
B_{t}(x, y, z)=\left(\begin{array}{cc}
1, \text { if } & V_{t}(x, y, z)>k \\
0, \text { if } & V_{t}(x, y, z) \leq k \cup V_{t}(x, y, z) \notin H
\end{array}\right.
$$

where $k$ is a scalar value that can be assigned a small value assuming the background intensity is zero. Then, we extract the object (head) from the background. $H$ is the set of slices representing head-only region, without the neck and shoulder structures. $H=\{$ All the head voxels $\}$;

Step 2) We resample, with a higher resolution (upsampling) the tilted volume $V_{t}$ in vertical axis, to make each voxel a cubic.

Let the original image matrix size be $N_{x} \times N_{y} \times N_{z}$ voxels, and the dimension of each voxel is $d_{x} \times d_{y} \times d_{z}$. We know that $d_{x}=d_{y}, d_{z}=\beta d_{x}$ where $\beta$ is a constant double value that denotes the ratio between the height of the voxel in $z$ direction and its horizontal dimension. The new dimension in $z$ is therefore written as $N_{z}$ ' where $N_{z}{ }^{\prime}=\left(d_{z} / d_{x}\right) N_{z}=\beta N_{z}$, such that each voxel dimension $d_{z}{ }^{\prime}=\left(d_{z} / \beta\right)=d_{x}=d_{y}$.

We interpolate $(\beta-1) N_{z} \times N_{x} \times N_{y}$ points from the existing sample $N_{z} \times N_{x} \times N_{y}$ points. We apply cubic B spline interpolation [9] since it provides high order estimation of the parameters to generate better visual appearance. Using this process, a series of unique cubic polynomials are fitted between each of the data points, with the expectation that the curve obtained be continuous and appear smooth.

We resample again, the volumetric data $B_{t}$ in $x, y, z$ axis, with a lower resolution (down-sampling), to reduce the computational throughput while maintain the closest 
solution to the optimum. The new dimension of the stack image slices is defined as $\bar{N}_{z} \times \bar{N}_{x} \times \bar{N}_{y}=(1 / u) N_{z}{ }^{\prime} \times N_{x} \times$ $N_{y}$, where $u$ is an integer, $u>1$, that preserves equally spaced samples in $x, y$ and $z$ dimensions every $u$ pixels

Step 3) The sampled object inside the sphere is processed by the algorithm that solves the eigenvalue problem associated with the object's inertia matrix. Firstly, we compute the centroid of the brain [1]. Let the centroid of the binary
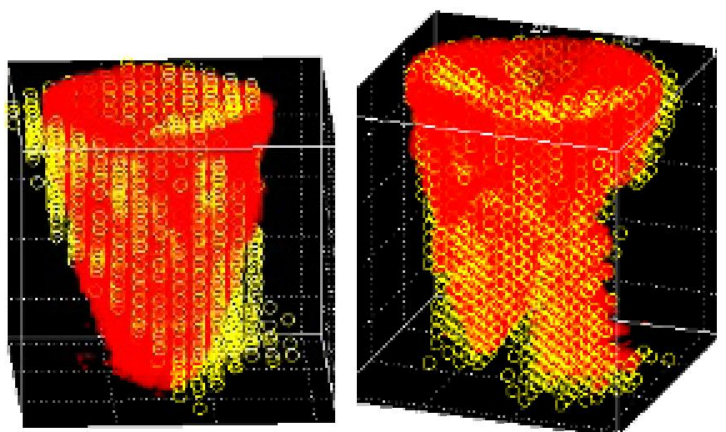

Fig. 3. The automatic correction of the titled volume (yellow dots). The red clouds depicts the corrected volume.

Volume $B_{t}(x, y, z)$ be represented by $\left(x_{g}, y_{g}, z_{g}\right)^{T}$ where

$$
\begin{aligned}
& x_{g}=\frac{\sum_{x, y, z} x B_{t}(x, y, z)}{\sum_{x, y, z} B_{t}(x, y, z)} \\
& y_{g}=\frac{\sum_{x, y, z} y B_{t}(x, y, z)}{\sum_{x, y, z} B_{t}(x, y, z)} \\
& z_{g}=\frac{\sum_{x, y, z} z B_{t}(x, y, z)}{\sum_{x, y, z} B_{t}(x, y, z)}
\end{aligned}
$$

We compute the covariance matrix $I$ of the volumetric binary image $B_{t}(x, y, z)$. The covariance matrix $I$ can be derived from the second-order central moments as follows:

$$
I=\left[\begin{array}{ccc}
I_{x x} & -I_{x y} & -I_{x z} \\
-I_{y x} & I_{y y} & I_{y z} \\
-I_{z x} & -I_{z y} & I_{z z}
\end{array}\right]
$$

where $I_{x x}=\sum_{x, y, z}\left[\left(y-y_{g}\right)^{2}+\left(z-z_{g}\right)^{2}\right] B_{i}(x, y, z)$

$$
\begin{aligned}
& I_{y y}=\sum_{x, y, z}\left[\left(x-x_{g}\right)^{2}+\left(z-z_{g}\right)^{2}\right] B_{t}(x, y, z) \\
& I_{z z}=\sum_{x, y, z}\left[\left(x-x_{g}\right)^{2}+\left(y-y_{g}\right)^{2}\right] B_{t}(x, y, z) \\
& I_{x y}=I_{y x}=\sum_{x, y, z}\left(x-x_{g}\right)\left(y-y_{g}\right) B_{t}(x, y, z) \\
& I_{x z}=I_{z x}=\sum_{x, y, z}\left(x-x_{g}\right)\left(z-z_{g}\right) B_{t}(x, y, z)
\end{aligned}
$$

$$
I_{y z}=I_{z y}=\sum_{x, y, z}\left(y-y_{g}\right)\left(z-z_{g}\right) B_{t}(x, y, z)
$$

The inertia matrix $J$ can be formed from covariance matrix $I, J=\operatorname{trace}(I) I_{0}-I$ where $I_{0}$ is the $3 \times 3$ identify matrix The three eigenvectors of $J$ are the principle axes, which are mutually orthogonal to each other. The centroid and the principal axes completely describe the orientation of a volume at an arbitrary orientation. The eigenvector corresponding to the smallest eigenvalue has the direction that is orthogonal to the mid-sagittal plane. Therefore, by computing the angle of this eigenvector with respect to the $x, y$ and $z$ axes, we actually acquire rotational angles(yaw, roll and pitch) of the mid-sagittal plane. See Fig. 1.

Step 4) Affine spatial transformation for tilt correction after the principle axes have been derived. let $R=R_{\alpha} R_{\beta} R_{\gamma}$ represent the rotation matrix as

$$
\begin{aligned}
& R_{\alpha} R_{\beta} R_{\gamma}= \\
& {\left[\begin{array}{ccc}
\cos (\alpha) & \sin (\alpha) & 0 \\
-\sin (\alpha) & \cos (\alpha) & 0 \\
0 & 0 & 1
\end{array}\right]\left[\begin{array}{ccc}
\cos (\beta) & 0 & -\sin (\beta) \\
0 & 1 & 0 \\
\sin (\beta) & 0 & \cos (\beta)
\end{array}\right]\left[\begin{array}{ccc}
1 & 0 & 0 \\
0 & \cos (\gamma) & \sin (\gamma) \\
0 & -\sin (\gamma) & \cos (\gamma)
\end{array}\right]}
\end{aligned}
$$

where $\alpha, \beta, \gamma$ are the rotation angles with respect to the $x$, $y$ and $z$ axes(Yaw, Roll, Pitch), respectively. Let $T$ represent the translation matrix.

$$
\mathrm{T}=\left[\begin{array}{cccc}
1 & 0 & 0 & 0 \\
0 & 1 & 0 & 0 \\
0 & 0 & 1 & 0 \\
\sigma \mathrm{x} & \sigma y & \sigma z & 1
\end{array}\right]
$$

where $\sigma x, \sigma y, \sigma z$ are the offset from the centroid of the head to the center of the scanner coordinates. $V_{c}=V_{t} \times R \times T$ where $V_{t}$ is the original input volume. $V_{c}$ is the $3 \mathrm{D}$ corrected volume re-centered at the centroid $\left(x_{g}, y_{g}, z_{g}\right)^{T}$. Re-slicing is conducted on the re-centered and re-tilted volume $V_{c}$. Again, we implement cubic spline interpolation to achieve better smoothness and higher precision. See Fig. 3 .

\section{RESULT}

In Fig. 4-6, we illustrate the method on different examples. We applied our method to brain MRI images from 15 patients selected from the pool of PACS (picture archiving and communication system) image database at Columbia Presbyterian Hospital. The dataset is composed of a mixture of T1-weighted MRI scans and T2-weighted MRI scans, half of which have distinctive brain lesion and certain level of brain shift. Each scan is represented by a matrix of dimension $256 \times 256 \times 124$, with voxel dimension $1.01 \times 1.01 \times 2.0 \mathrm{~mm}^{3}$

For each patient, two image volumes are generated, one that has matrix dimension of $256 \times 256 \times 248$, and the other is $128 \times 128 \times 124$. A total of 30 image volumes were 
generated and qualitatively assessed by a physician. Out of 30,29 cases were judged to be highly accurate in terms of head tilt correction and re-interpolation of the brain along the $\mathrm{z}$-axis. A prospective study for quantitative validation is currently being designed. One set of result has been illustrated in Fig. 7, this patient has a large brainstem lesion with ventricular effacement and left-to-right shift. However, the local asymmetry doesn't modify the global orientation of the brain. The result shows that we can still precisely capture the symmetry plane regardless of the local pathological conditions.

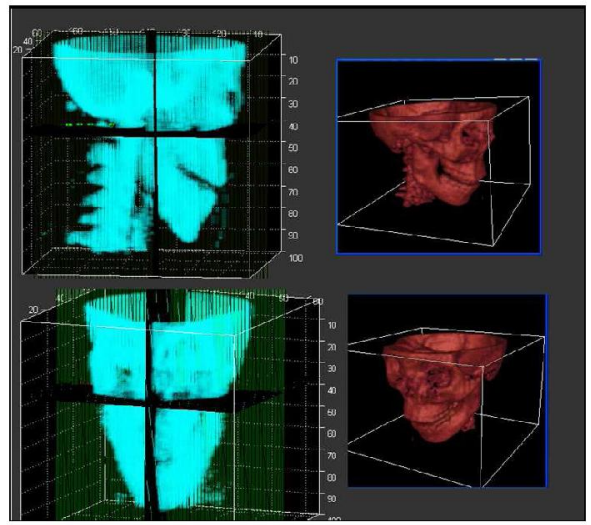

Fig.4. This figure demonstrates when the CT brain images are loaded into the program(on the right column), our program automatically computes 3 planes that are orthogonal to the 3 distinctive principle axes(on the left column).

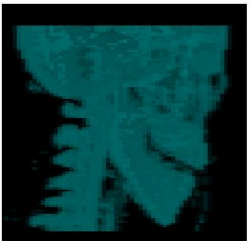

(a)

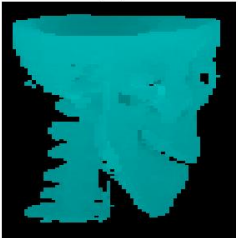

(d)

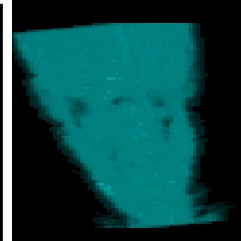

(b)

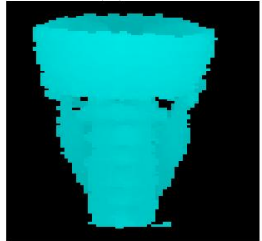

(e)

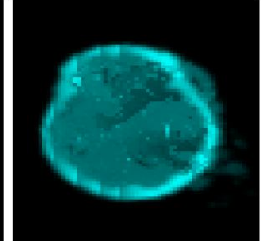

(c)

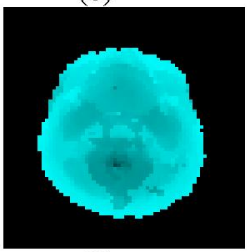

(f)
Fig. 5. (a)-(c) CT scans with tilted volume. (d)-(f) corresponding rerendered volume after correction of the tilt.

\section{CONCLUSION}

In this paper, an automatic, cost-effective method to automatically correct the tilt of the brain in $3 \mathrm{D}$ space is presented. The technique provides a new tool for symmetry based analysis of the brain pathologies, which present as asymmetric features (e.g. Tumor, stroke, bleeding). Preliminary results for MR and CT brain scans have shown promise and further studies to validate the method are ongoing. As was demonstrated, the technique performs robustly even in the presence of acquisition noise, bias field and pathological asymmetry because the method exams the global geometrical property of the head by the principle component analysis. When the data is truncated or the field of view is neck/shoulder-inclusive, the assumption that the head is ellipsoid-like $3 \mathrm{D}$ object is not met and the technique may fail. Failure of the technique in these cases can be avoided by the correct inclusion or exclusion of an object of interest. We continue working on an improvement of the method that can be robust and will accommodate a wide range of challenging cases generated by an imperfect acquisition process of head/brain imaging.

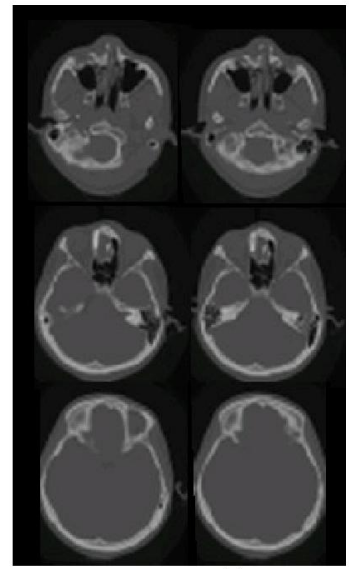

(a)

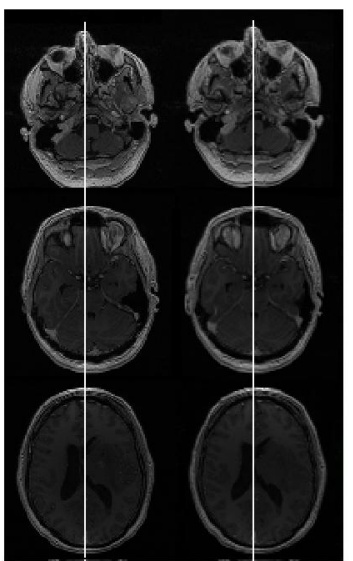

(b)
Fig. 6. (a) Left column: misaligned CT scans; right column: corrected CT scans. (b) Left column: misaligned MR scans; right column: corrected MRI scans.

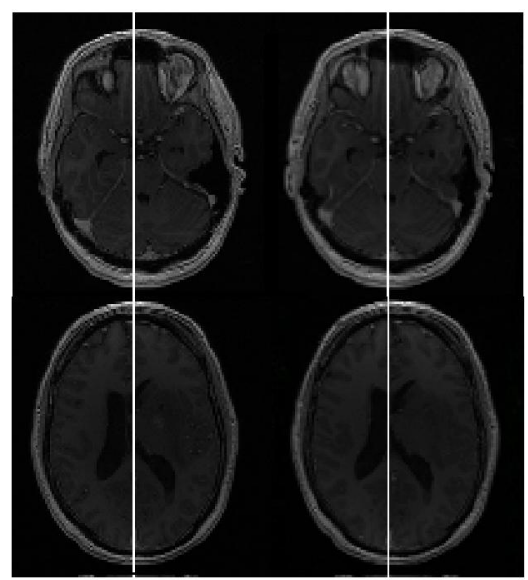

Fig.7. MR images with big brain lesion and certain level of brain shift. Left column: original images; Right column: images in the volume with corrected tilt.

\section{ACKNOWLEDGEMENTS}


This work has been supported, in part, by Department of Neurosurgery at Columbia University.

\section{REFERENCES}

[1] Atam P.Dhawan, "Image registration", book chapter in "Medical Image Analysis", p254-p259, published by John Wiley \& Sons, 2003

[2] Sylvain Prima, Sébastien Ourselin, and Nicholas Ayache, "Computation of the Mid-Sagittal Plane in 3-D Brain Images",IEEE transaction on medical imaging, vol.21, no.2, february 2002

[3] Celina Imielinska, Xin Liu, Joel Rosiene et al., "Towards Objective Quantification of Perfusion-Weighted Computed Tomography in the Setting of Subarachnoid Hemorrhage: Quantification of Symmetry and Automated Delineation of Vascular Territories ", Journal of Academic Radiology ,2005

[4] M. E. Brummer, "Hough transform detection of the longitudinal fissure in tomographic head images," IEEE Trans. Med. Imag., vol. 10, pp. 74 81, Mar. 1991.

[5] B. A. Ardekani, J. Kershaw, M. Braun, and I. Kanno, "Automatic detection of the mid-sagittal plane in 3-D brain images," IEEE Trans. Med Imag., vol. 16, pp. 947-952, Dec. 1997.

[6] C. Sun and J. Sherrah, "3D symmetry detection using the extended gaussian image," IEEE Trans. Pattern Anal. Machine Intell., vol. 19, pp. 164-168, Feb 1997.

[7] Y. Liu, R. T. Collins, and W. E. Rothfus, "Robust Midsagittal Plane Extraction from Normal and Pathological 3D Neuroradiology Images" IEEE Transactions on Medical Imaging, Vol. 20, No. 3, March, 2001, pp. $175-192$

[8] P. Minovic, S. Ishikawa, K. Kato, "Symmetry Identification of a 3D Object Represented by Octree", IEEE Transactions on Pattern Analysis and Machine Intelligence, v.15 n.5, p.507-514, May 1993

[9] J.K. Udupa and G.T. Herman "3D Imaging in Medicine: Second Edition", CRC Press, 1998

[10] P. C. Marais, R. Guillemaud, M. Sakuma, A. Zisserman, and M. Brady, "Visualising cerebral asymmetry," in Lecture Notes in Computer Science,K. H. Höhne and R. Kikinis, Eds, Hamburg, Germany: Springer,Sept. 1996, vol. 